International Mathematical Forum, 5, 2010, no. 21, 1025 - 1036

\title{
An Explicit Method for Solving Fuzzy Partial Differential Equation
}

\author{
A. Farajzadeh \\ Islamic Azad university, Kermanshah Branch, Iran \\ faraj1348@yahoo.com \\ A. Hossein Pour \\ Islamic Azad university, Kermanshah Branch, Iran \\ hoseinpour.azadeh@gmail.com \\ M. Amini \\ Department of Mathematics, Payam Noor University (PNU) \\ Songhor, Kermanshah, Iran \\ Mamini1356@yahoo.com
}

\begin{abstract}
This paper deals with a numerical method for the solution of the fuzzy heat equation. First we express the necessary materials and definitions, then consider a difference scheme for the one dimensional heat equation. In forth section we express the necessary conditions for stability and check stability of our scheme. In final part we give an example for consider numerical results. In this example we obtain the Hausdorff distance between exact solution and approximate solution.
\end{abstract}

Keywords: Fuzzy numbers, Fuzzy heat equation, Finite difference scheme, stability 


\section{Introduction}

The topics of numerical methods for solving fuzzy differential equations have been rapidly growing in recent years. The concept of fuzzy derivative was first introduced by Chang and Zadeh in [10]. It was following up by Dubois and Prade in [2], who defined and used the extension principle. Other methods have been discussed by Puri and Relescu in [4] and Goetschel and Voxman in [9]. The initial value problem for first order fuzzy differential equations have been studied by several authors $[7,5,6,8,11]$. on the metric space $\left(E^{n}, D\right)$ of normal fuzzy convex sets with the distance $D$ given by the maximum of the Hausdorff distances between the corresponding level sets.

\section{Materials and definitions}

We begin this section with defining the notation we will use in the paper. We let $X$ is a collection of objects denoted generically by $x$, then a fuzzy set $\widetilde{A}$ in $X$ is a set of ordered pairs:

$$
\widetilde{A}=\left\{\left(x, \mu_{\widetilde{A}}(x)\right) \mid x \in X\right\}
$$

$\mu_{\widetilde{A}}$ is called the membership function or grade of membership of $x$ in $\widetilde{A}$. The range of the membership function is a subset of the nonnegative real numbers whose supremum is finite.

Definition 2.1. The set of elements that belong to the fuzzy set $\widetilde{A}$ at least to the degree $\alpha$ is called the $\alpha$-cut set:

$$
A_{\alpha}=\left\{x \in X \mid \mu_{\widetilde{A}}(x) \geq \alpha\right\}
$$

$A_{\alpha}^{\prime}=\left\{x \in X \mid \mu_{\widetilde{A}}(x)>\alpha\right\}$ is called strong $\alpha$-cut.

Definition 2.2. The triangular fuzzy number $\widetilde{N}$ is defined by three numbers $\alpha<m<\beta$ as fllows:

$$
\widetilde{A}=(\alpha, m, \beta)
$$




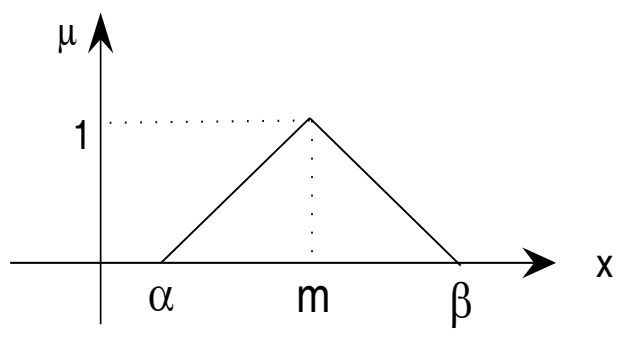

Fig 1.

This representation is interpreted as membership function(Fig 1):

$$
\mu_{\widetilde{A}}(x)=\left\{\begin{array}{cc}
\frac{x-\alpha}{m-\alpha} & \alpha \leq x<m \\
1 & x=m \\
\frac{x-\beta}{m-\beta} & m<x \leq \beta \\
0 & \text { o.w }
\end{array}\right.
$$

If $\alpha>0(\alpha \geq 0)$ then $\widetilde{A}>0(\tilde{A} \geq 0)$, If $\beta<0(\beta \leq 0)$ then $\widetilde{A}<0(\widetilde{A} \leq 0)$.

Definition 2.3. An arbitrary fuzzy number is showed by an ordered pair of functions $(\underline{a}(r), \bar{a}(r)), 0 \leq r \leq 1$, which satisfies the following requirements:

1. $\underline{a}(r)$ is a bounded left semicontinuous non-decreasing function over $[0,1]$,

2. $\bar{a}(r)$ is a bounded left semicontinuous non-increasing function over $[0,1]$,

3. $\underline{a}(r) \leq \bar{a}(r), 0 \leq r \leq 1$.

In particular, if $\underline{a}, \bar{a}$ are linear functions we have a triangular fuzzy number.

A crisp number $a$ is simply represented by $\underline{a}(r)=\bar{a}(r)=a, 0 \leq r \leq 1$.

Definition 2.4. For arbitrary fuzzy numbers $u=(\underline{u}(r), \bar{u}(r)), v=(\underline{v}(r), \bar{v}(r))$ we have algebraic operations bellow:

1. $k u= \begin{cases}(k \underline{u}, k \bar{u}) & k \geq 0 \\ (k \bar{u}, k \underline{u}) & k<0\end{cases}$ 
2. $u+v=(\underline{u}(r)+\underline{v}(r), \bar{u}(r)+\bar{v}(r))$

3. $u-v=(\underline{u}(r)-\bar{v}(r), \bar{u}(r)-\underline{v}(r))$

4. $u \cdot v=($ mins, $\operatorname{maxs})$, which

$$
s=\{\underline{u} \underline{v}, \underline{u} \bar{v}, \bar{u} \underline{v}, \bar{u} \bar{v}\}
$$

Remark. Since the $\alpha$-cut of fuzzy numbers is always a closed and bounded interval, so we can write $\widetilde{A}_{\alpha}=[\underline{a}(\alpha), \bar{a}(\alpha)]$, for all $\alpha$.

Definition 2.5. Assume $u=(\underline{u}(r), \bar{u}(r)), v=(\underline{v}(r), \bar{v}(r))$ are two fuzzy numbers. The Hausdorff metric $D_{H}$ is defined by:

$$
D_{H}(u, v)=\sup _{r \in[0,1]} \max \{|\underline{u}(r)-\underline{v}(r)|,|\bar{u}(r)-\bar{v}(r)|\}
$$

This metric is a bound for error. By it we obtain the difference between exact solution and approximate solution.

\section{Finite difference method}

In this section we solve the fuzzy heat equation by an explicit method. Assume $\widetilde{U}$ is a fuzzy function of the independent crisp variables $x$ and $t$. We define:

$$
I=\{(x, t) \mid 0 \leq x \leq 1,0 \leq t \leq T\}
$$

A $\alpha$-cut of $\widetilde{U}(x, t)$ and its the parametric form, will be:

$$
\widetilde{U}(x, t)[\alpha]=[\underline{U}(x, t ; \alpha), \bar{U}(x, t ; \alpha)] .
$$

We let that the $\underline{U}(x, t ; \alpha), \bar{U}(x, t ; \alpha)$ have continuous partial differential, therefor $\left(D_{t}-\right.$ $\left.a^{2} D_{x}^{2}\right) \bar{U}(x, t ; \alpha)$, and $\left(D_{t}-a^{2} D_{x}^{2}\right) \underline{U}(x, t ; \alpha)$ are continuous for all $(x, t) \in I$, all $\alpha \in[0,1]$. Now we consider heat equation

$$
\left(D_{t}-a^{2} D_{x}^{2}\right) \widetilde{U}=\widetilde{0}
$$

With boundary conditions and initial condition:

$$
\left\{\begin{array}{l}
\widetilde{U}(0, t)=\widetilde{U}(1, t)=\widetilde{0} \\
\widetilde{U}(x, 0)=\widetilde{f}(x)
\end{array}\right.
$$




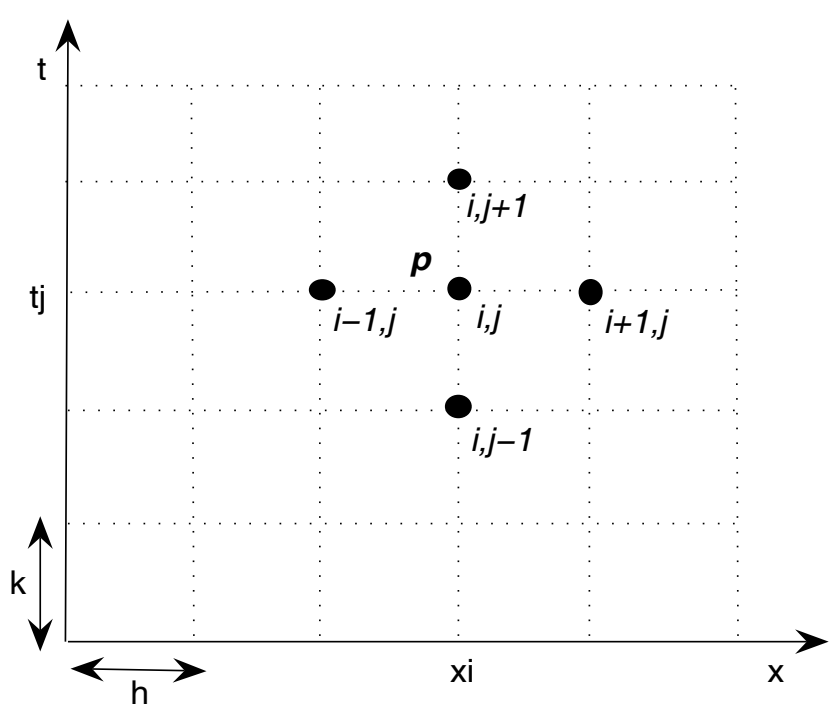

Fig 2.

We divide the domain $[0,1] \times[0, T]$ in to $M \times N$ mesh with spatial step size $h=\frac{1}{N}$ in $x$-direction and $k=\frac{T}{M}$ in $t$-direction. The gride points are given by (Fig 2):

$$
\begin{array}{cc}
x_{i}=i h & i=0,1, \cdots, N \\
t_{j}=j k & j=0,1, \cdots, M
\end{array}
$$

Denote the value of $\widetilde{U}$ at the representative mesh point $p\left(x_{i}, t_{j}\right)$ by:

$$
\widetilde{U}_{p}=\widetilde{U}\left(x_{i}, t_{j}\right)=\widetilde{U}_{i, j}
$$

and also parametric form of fuzzy number $\widetilde{U}_{i, j}$ is:

$$
\widetilde{U}_{i, j}=\left(\underline{U}_{i, j}, \bar{U}_{i, j}\right)
$$

We have:

$$
\left\{\begin{array}{l}
\left(D_{t}\right) \widetilde{U}_{i, j}=\left(\underline{D_{t} U_{i, j}}, \overline{D_{t} U_{i, j}}\right) \\
\left.\left(D_{x}^{2}\right) \widetilde{U}_{i, j}=\underline{\left(D_{x}^{2} U_{i, j}\right.}, \overline{D_{x}^{2} U_{i, j}}\right)
\end{array}\right.
$$

Then by taylor's expansion we obtain:

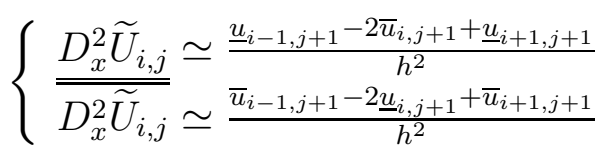


And also for $\left(D_{t}\right) \widetilde{U}$ at $p$, we have:

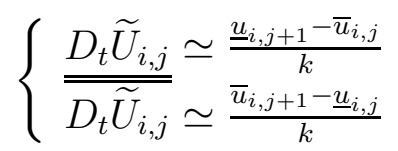

Parametric form of heat equation will be:

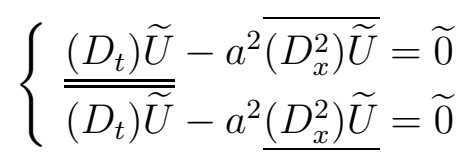

By (4) and (5) the difference scheme for heat equation is:

$$
\left\{\begin{array}{l}
\frac{\underline{u}_{i, j+1}-\bar{u}_{i, j}}{k}-a^{2} \frac{\bar{u}_{i-1, j+1}-2 \underline{u}_{i, j+1}+\bar{u}_{i+1, j+1}}{h^{2}}=0 \\
\frac{\bar{u}_{i, j+1}-\underline{u}_{i, j}}{k}-a^{2} \frac{\underline{u}_{i-1, j+1}-2 \bar{u}_{i, j+1}+\underline{u}_{i+1, j+1}}{h^{2}}=0
\end{array}\right.
$$

By above equations we obtain:

$$
\left\{\begin{array}{l}
-r \underline{u}_{i-1, j+1}+(1+2 r) \bar{u}_{i, j+1}-r \underline{u}_{i+1, j+1}=\underline{u}_{i, j} \\
-r \bar{u}_{i-1, j+1}+(1+2 r) \underline{u}_{i, j+1}-r \bar{u}_{i+1, j+1}=\bar{u}_{i, j}
\end{array}\right.
$$

Where:

$$
r=\frac{k a^{2}}{h^{2}}
$$

$\widetilde{U}=(\underline{u}, \bar{u})$ is the exact solution of the approximating difference equations, and $x_{i},(i=$ $0,1, \cdots, N)$ and $t_{j},(j=0,1, \cdots, M)$.

Since the boundary values are know at $x_{0}$ and $x_{N}$, we have $2(N-1)$ equations with $2(N-1)$ unknow. Therefore equations can be written in matrix as:

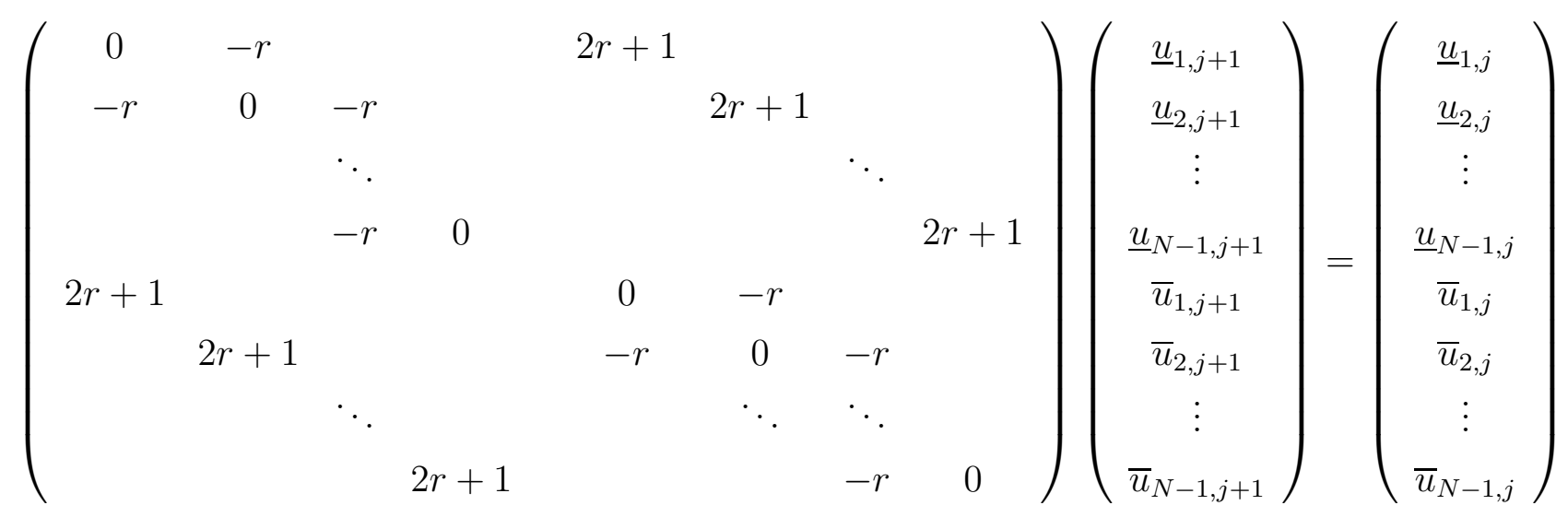


Then we will have:

$$
A\left(\begin{array}{c}
\underline{U}_{j+1} \\
\bar{U}_{j+1}
\end{array}\right)=\left(\begin{array}{c}
\underline{U}_{j} \\
\bar{U}_{j}
\end{array}\right) \Rightarrow\left(\begin{array}{c}
\underline{U}_{j+1} \\
\bar{U}_{j+1}
\end{array}\right)=A^{-1}\left(\begin{array}{c}
\underline{U}_{j} \\
\bar{U}_{j}
\end{array}\right)
$$

Where:

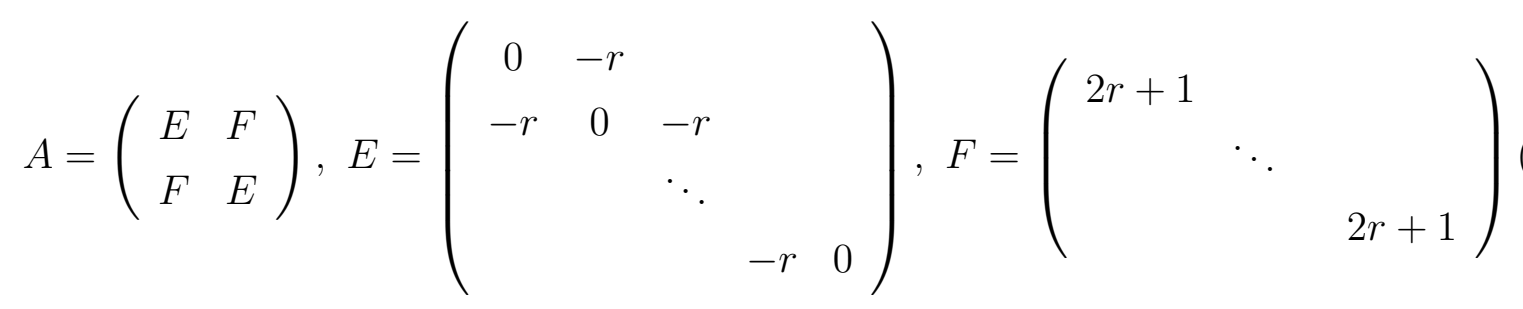

\section{Stability of fuzzy heat equation}

Definition 4.1. The largest of the eigenvalues of matrix $A$, is showed by $\rho(A)$.

Remark 4.1. The necessary and sufficient condition for the difference equations to be stable is $\rho(A) \leq 1[3]$.

Remark 4.2. If $A^{-1}$ be inverse of matrix $A$, then $\rho\left(A^{-1}\right)=\frac{1}{\rho(A)}[1]$.

Remark 4.3. The eigenvalues of a $N \times N$ tridiagonal matrix

$$
\left(\begin{array}{cccccc}
a & b & & & & \\
c & a & b & & & \\
& \ddots & \ddots & \ddots & & \\
& & & c & a & b \\
& & & & c & a
\end{array}\right)
$$

are

$$
\lambda_{s}=a+2 \sqrt{b c} \cos \frac{s \pi}{N+1}, \quad s=1, \cdots, N \quad[3]
$$

Theorem 4.1. Let matrix $A$ has spacial structure as follow

$$
A=\left(\begin{array}{ll}
E & F \\
F & E
\end{array}\right)
$$


then the eigenvalues of $A$ are union of eigenvalues of $E+F$ and eigenvalues of $E-F[12]$.

Now for demonstration stability of our difference scheme is sufficient to show in (11) $\rho\left(A^{-1}\right)<1$. Thus by theorem (4.1) we find eigenvalues of $E+F$ and $E-F$. We have

$$
E+F=\left(\begin{array}{cccc}
1+2 r & -r & & \\
-r & 1+2 r & -r & \\
& \ddots & \ddots & \\
& & -r & 1+2 r
\end{array}\right)
$$

and

$$
E-F=\left(\begin{array}{cccc}
-1-2 r & -r & & \\
-r & -1-2 r & -r & \\
& \ddots & \ddots & \\
& & -r & -1-2 r
\end{array}\right)
$$

We obtain

$$
\begin{array}{r}
E+F=I+r S, \\
E-F=-I-r S^{\prime}
\end{array}
$$

Where

$$
S=\left(\begin{array}{cccc}
2 & -1 & & \\
-1 & 2 & -1 & \\
& \ddots & \ddots & \\
& & -1 & 2
\end{array}\right), S^{\prime}=\left(\begin{array}{cccc}
2 & 1 & & \\
1 & 2 & 1 & \\
& \ddots & \ddots & \\
& & 1 & 2
\end{array}\right)
$$

Thus

$$
\lambda_{s}=\lambda_{s^{\prime}}=2+2 \cos \frac{k \pi}{N}=4 \cos ^{2} \frac{k \pi}{2 N}, \quad k=1,2, \cdots, N-1
$$

Hence the eigenvalues of $E+F$ and $E-F$ are

$$
\begin{gathered}
\lambda_{E+F}=1+4 r \cos ^{2} \frac{k \pi}{2 N}, \quad k=1,2, \cdots, N-1 \\
\lambda_{E-F}=-1-4 r \cos ^{2} \frac{k \pi}{2 N}, \quad k=1,2, \cdots, N-1
\end{gathered}
$$


we know

$$
\begin{array}{r}
\rho(E+F)=\max _{k}\left|1+4 r \cos ^{2} \frac{k \pi}{2 N}\right|, \quad k=1,2, \cdots, N-1 \\
\rho(E-F)=\max _{k}\left|-1-4 r \cos ^{2} \frac{k \pi}{2 N}\right|, \quad k=1,2, \cdots, N-1
\end{array}
$$

Since $\rho(E+F)=\rho(E-F)$ thus

$$
\rho(A)=\max _{k}\left|1+4 r \cos ^{2} \frac{k \pi}{2 N}\right|,
$$

and

$$
\rho\left(A^{-1}\right)=\frac{1}{\max \left|1+4 r \cos ^{2} \frac{k \pi}{2 N}\right|}<1, \quad \forall r>0 .
$$

Therefore our difference scheme is unconditionally stable.

\section{$5 \quad$ Numerical example}

In this section we test the proposed difference method on an example, whose exact solution is know to us.

Consider the fuzzy heat equation

$$
\frac{\partial \widetilde{U}}{\partial t}(x, t)=4 \frac{\partial^{2} \widetilde{U}}{\partial x^{2}}(x, t), \quad 0<x<1, t>0
$$

Subject to the boundary conditions and the initial condition

$$
\begin{gathered}
\widetilde{U}(0, t)=\widetilde{U}(1, t)=0, \quad t>0 \\
\widetilde{U}(x, 0)=\widetilde{f}(x)=\frac{2}{\pi} \widetilde{K} \sin \pi x
\end{gathered}
$$

and $\widetilde{K}[\alpha]=[\underline{k}(\alpha), \bar{k}(\alpha)]=[\alpha-1,1-\alpha]$. Which is easily seen to have exact solution for

$$
\begin{aligned}
& \frac{\partial \underline{U}}{\partial t}(x, t ; \alpha)=4 \frac{\partial^{2} \underline{U}}{\partial x^{2}}(x, t ; \alpha) \\
& \frac{\partial \bar{U}}{\partial t}(x, t ; \alpha)=4 \frac{\partial^{2} \bar{U}}{\partial x^{2}}(x, t ; \alpha)
\end{aligned}
$$

are

$$
\begin{aligned}
& \underline{U}(x, t ; \alpha)=\frac{2}{\pi} \underline{k}(\alpha) e^{-4 \pi^{2} t} \sin \pi x \\
& \bar{U}(x, t ; \alpha)=\frac{2}{\pi} \bar{k}(\alpha) e^{-4 \pi^{2} t} \sin \pi x
\end{aligned}
$$




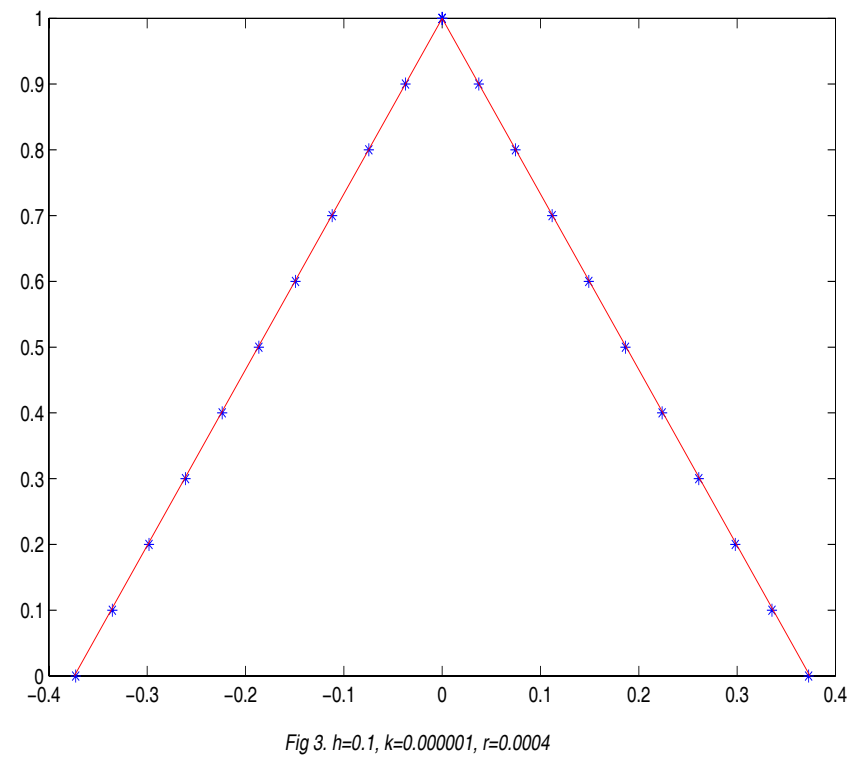

The exact and approximate solutions are shown in Figure (3) at the point $(0.2,0.00099)$ with $h=0.1, k=0.00001$ and in Figure(4) at the point $(0.02,0.0099)$ with $h=0.01, k=$ 0.0001 .

The Housdorff distance between solutions in first case is 0.0014 and in second case is 0.0015 .

\section{Conclusions}

Our purpose in this article is solving fuzzy partial differential equation (FPDE). We presented an explicit method for solving this equation, and we considered necessary conditions for stability of this method. In last section we given an example for consider numerical results. Also we compared the approximate solution and exact solution. Then we obtained the Hausdorf distance between them in two case. 


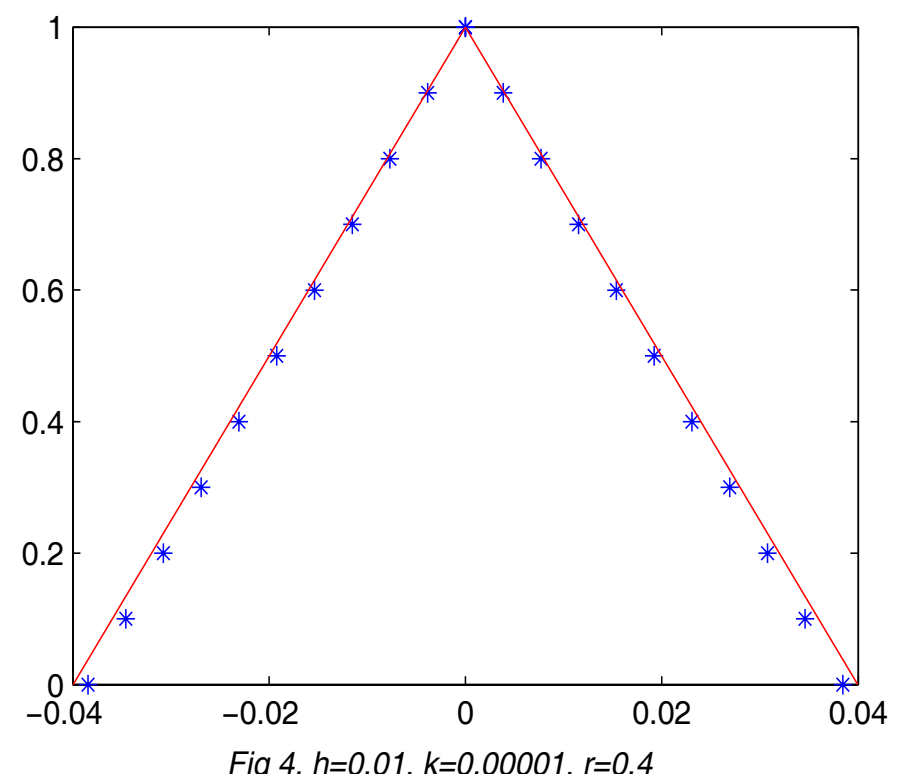

\section{References}

[1] Biswa Nath Data, Numerical Linear Algebra and Applications.

[2] D. Dubois and H. Prade, Towards fuzzy differential calculus: part 3, differentiation, Fuzzy Sets and Systems, 8 (1982) 225-233.

[3] G. D. Smith, Numerical solution of partial differential equations, (1993).

[4] M. L. Puri and D. A. Ralescu, Differentials of fuzzy functions, J. Math. Anal. Appl, 91 (1983) 321-325.

[5] O. Kaleva, Fuzzy differential equations, Fuzzy Sets and Systems, 24 (1987) 301-307.

[6] O. Kaleva, The cauchy problem for fuzzy differential equations, Fuzzy sets and systems, 35 (1990) 389-396.

[7] P. Diamond and P. Kloeden, Metric Spaces of Fuzzy Sets. World Scientific, Singapore, (1994).

[8] P. E. Kloeden, Remarks on Peano-like theorems for fuzzy differential equations, Fuzzy Sets and Systems, 44 (1991) 161-163. 
[9] R. Goetschel and W. Voxman, Elementary fuzzy calculus, Fuzzy sets and Systems, 18 (1988) 31-43.

[10] S. L. Chang and L.A. Zadeh, On fuzzy mapping and control, IEEE Trans, Systems Mah Cybemet, 2 (1972) 30-34.

[11] S. Seikkala, On the fuzzy initial value problem, Fuzzy Sets and Systems, 24 (1987) 319-330.

[12] T. Allahviranloo and N. Ahmadi and E. Ahmadi and Kh. Shams Alketabi, Block Jacobi two-stage method for fuzzy systems of linear equations, Applied Mathematics and Computation, 175 (2006) 1217-1228.

Received: June, 2009 